\title{
Hemoglobin quantification in red blood cells via dry mass mapping based on UV absorption
}

\author{
Nischita Kaza $\odot,{ }^{a}$ Ashkan Ojaghi $\odot$, ${ }^{b}$ and Francisco E. Robles ${ }^{\text {b, } *}$ \\ ${ }^{a}$ Georgia Institute of Technology, School of Electrical and Computer Engineering, \\ Atlanta, Georgia, United States \\ ${ }^{\mathrm{b}}$ Georgia Institute of Technology and Emory University, Wallace H. Coulter \\ Department of Biomedical Engineering, Atlanta, Georgia, United States
}

\begin{abstract}
Significance: The morphological properties and hemoglobin $(\mathrm{Hb})$ content of red blood cells (RBCs) are essential biomarkers to diagnose or monitor various types of hematological disorders. Label-free mass mapping approaches enable accurate $\mathrm{Hb}$ quantification from individual cells, serving as promising alternatives to conventional hematology analyzers. Deep ultraviolet (UV) microscopy is one such technique that allows high-resolution, molecular imaging, and absorption-based mass mapping.

Aim: To compare UV absorption-based mass mapping at four UV wavelengths and understand variations across wavelengths and any assumptions necessary for accurate $\mathrm{Hb}$ quantification.

Approach: Whole blood smears are imaged with a multispectral UV microscopy system, and the RBCs' dry masses are computed. This approach is compared to quantitative phase imagingbased mass mapping using data from an interferometric UV imaging system.

Results: Consistent $\mathrm{Hb}$ mass and mean corpuscular $\mathrm{Hb}$ values are obtained at all wavelengths, with the precision of the single-cell mass measurements being nearly identical at 220, 260, and $280 \mathrm{~nm}$ but slightly lower at $300 \mathrm{~nm}$.

Conclusions: A full hematological analysis (including white blood cell identification and characterization, and $\mathrm{Hb}$ quantification) may be achieved using a single UV illumination wavelength, thereby improving the speed and cost-effectiveness.

(C) The Authors. Published by SPIE under a Creative Commons Attribution 4.0 Unported License. Distribution or reproduction of this work in whole or in part requires full attribution of the original publication, including its DOI. [DOI: 10.1117/1.JBO.26.8.086501]
\end{abstract}

Keywords: deep-UV microscopy; mass mapping; quantitative phase imaging; red blood cells; hemoglobin; hematology analysis.

Paper 210112LR received Apr. 14, 2021; accepted for publication Jul. 16, 2021; published online Aug. 10, 2021.

\section{Introduction}

The morphological and molecular properties of blood cells are important indicators of health and are diagnostic markers for several diseases. ${ }^{1-7}$ The morphology, size, and hemoglobin $(\mathrm{Hb})$ content of red blood cells (RBCs) serve as essential biomarkers to diagnose or monitor various types of anemias and other diseases, including malaria and thalassemia. ${ }^{5-7} \mathrm{RBCs}$ are enucleated, terminally differentiated cells that are composed of $95 \%$ to $98 \% \mathrm{Hb}$ by dry mass (mass of all the constituents of a cell in the absence of water).$^{8-10}$ Thus, the dry mass of RBCs is of clinical significance, and hematology analyzers routinely used to obtain a complete blood count (CBC) from peripheral blood samples measure indices such as the dry mass of $\mathrm{Hb}$ in the form of the mean corpuscular $\mathrm{Hb}(\mathrm{MCH})$, and the mean corpuscular $\mathrm{Hb}$ concentration. ${ }^{7}$ Along with mean values, single-cell measurements are invaluable to completely characterize a sample's distribution. ${ }^{11,12}$ In many clinical hematology analyzers, $\mathrm{Hb}$ concentration is obtained from the optical scattering of spherical cells. ${ }^{13}$ Owing to the characteristic biconcave shape of RBCs, they have to be treated with a sphering agent, which lengthens the procedure and leaves cells in a

*Address all correspondence to Francisco E. Robles, robles@gatech.edu 
nonphysiological state. ${ }^{14,15}$ In addition, in some pathological conditions, such as sickle cell anemia, the RBCs are difficult to sphere. ${ }^{16}$

Label-free optical techniques to quantify the dry mass and other physical properties of individual cells ${ }^{10,15,17-22}$-in their natural state without requiring additional pretreatment or sample preparation —offer an excellent alternative and have been successfully applied to quantify $\mathrm{Hb}$ in RBCs. ${ }^{5,6,15,21-24}$ For example, quantitative phase imaging (QPI)-based methods ${ }^{5,6,17-19,21,23}$ measure the phase accumulated in biological samples and translate this metric into dry mass density by leveraging the linear relationship between refractive index and mass concentration. ${ }^{17}$ QPI methods gained popularity due to their accuracy, sensitivity, and speed, but they lack molecular specificity and cannot clearly distinguish between the different organelles or cellular compartments having different chemical compositions. ${ }^{25}$

Deep-ultraviolet (UV) microscopy has emerged as a high-resolution, label-free imaging technique capable of providing quantitative molecular information due to the distinctive absorptive properties of endogenous biomolecules in this region of the spectrum..$^{20,24,26-28}$ Deep-UV microscopy images have been used to successfully generate accurate nucleic acid and protein mass maps of live cells owing to their intrinsic molecular contrast, without the need for laborious fixing and staining procedures, ${ }^{10,20,22,26}$ we recently demonstrated that deep-UV microscopy images acquired at $300 \mathrm{~nm}$ could accurately measure $\mathrm{Hb}$ mass. ${ }^{24}$ Moreover, a five-part white blood cell (WBC) differential count was achieved by analyzing absorption and morphological features at $260 \mathrm{~nm}$, and a colorization scheme was devised, which could recapitulate the goldstandard Giemsa stained appearance of blood cells using images acquired at 260, 280, and $300 \mathrm{~nm}$.

UV absorption-based mass mapping has multiple advantages over QPI-based methods. First, UV mass maps have higher spatial resolution than typical QPI-based measurements due to the UV light's shorter wavelength. In addition, while a number of QPI systems rely on an interferometric setup to measure the phase, intensity-based UV imaging systems can have simpler instrumentation (they do not require beam splitters, gratings, etc.) and have better light throughput (i.e., they do not have to split the light source to obtain a reference field). Most importantly, UV absorption-based mass mapping is able to distinguish and quantify the different chemical constituents of a cell rather than grouping them together, as in QPI-based mass maps.

The accuracy of UV-absorption-based $\mathrm{Hb}$ mass mapping depends on the wavelength, and oxygenation-state dependent absorption properties of this molecule. In this letter, we present a comparison of RBC mass mapping, and thereby $\mathrm{Hb}$ quantification, at four UV wavelengths: 220, 260, 280, and $300 \mathrm{~nm}$ using transmission microscopy images from our multispectral UV imaging system. ${ }^{24}$ Note that 220 and $280 \mathrm{~nm}$ correspond to regions of high protein absorption, $260 \mathrm{~nm}$ corresponds to the absorption peak of nucleic acids, whereas absorption at $300 \mathrm{~nm}$ is only significant for $\mathrm{Hb}$. Our previous study ${ }^{24}$ leveraged this insight and used $260 \mathrm{~nm}$ for WBC identification and $300 \mathrm{~nm}$ for $\mathrm{Hb}$ analysis. However, imaging protocols could be greatly simplified if only a single wavelength was necessary to achieve both WBC and RBC (and thus $\mathrm{Hb}$ ) characterization.

In addition to comparing data acquired at four different UV wavelengths, we also compare our results to measurements obtained from QPI-based mass mapping using interferometric data from our UV hyperspectral interferometric (UHI) microscopy system. ${ }^{26}$ We demonstrate consistent hemoglobin mass quantification at all wavelengths and obtain $\mathrm{MCH}$ values lying within the normal reference range $(29.5 \pm 2.5 \mathrm{pg}){ }^{7}$ The single-cell mass measurements from UV absorption-based mass maps have the highest precision (measured in terms of the standard deviation) at $220 \mathrm{~nm}$, closely followed by 260 and $280 \mathrm{~nm}$ and slightly lower at $300 \mathrm{~nm}$. These results indicate that a full hematological analysis may be achieved using a single UV illumination wavelength.

\section{Materials and Methods}

The multispectral UV microscopy system depicted in Fig. S1 in Supplemental Material uses a laser-driven plasma source (EQ-99X LDLS, Energetiq Technology) that is relayed to the sample through an off-axis parabolic mirror (Newport Corporation) and a short-pass dichroic mirror 
(Thorlabs). A filter wheel fitted with UV band-pass filters $(\sim 10 \mathrm{~nm}$ FWHM, Chroma Technology) enables multispectral imaging at four center wavelengths: 220, 260, 280, and $300 \mathrm{~nm}$. A 40×, 0.5 NA UV-objective (LMU-40X, Thorlabs) is used for imaging, which provides an average spatial resolution of $\sim 280 \mathrm{~nm}$. The images at each wavelength are captured using a UV-sensitive CCD camera (pco.UV, PCO AG). We note that commercially available LEDs at 260, 280, and $300 \mathrm{~nm}$ can replace the broadband source and filter wheel, reducing the cost, complexity, and footprint of our system.

We compare the results from UV absorption-based mass mapping with QPI-based mass mapping using data acquired from our UHI microscopy system ${ }^{26}$ shown in Fig. S2 in Supplemental Material. This system uses a $4 \mathrm{f}$ interferometric configuration in a Mach-Zehnder geometry with the same light source, to enable coherent detection of optical fields. ${ }^{26}$ The same $40 \times \mathrm{UV}$ objective is used for imaging but data are collected with an imaging spectrometer (IsoPlane-160, Princeton Instruments) equipped with a back-illuminated sCMOS camera (Kuro 1200, Princeton Instruments). One spatial dimension of the sample is captured along the rows of the camera, whereas its spectrum is recorded along the columns. The sample is then line-scanned using a high-precision motorized stage (MLS2031, Thorlabs) to obtain a hyperspectral data-cube. The interferometric configuration and the use of beam splitters limit the light-throughput and do not allow imaging at wavelengths of interest below $240 \mathrm{~nm}$ where the source power is low (e.g., at $220 \mathrm{~nm}$, where all proteins have significant absorption). Thus, this system enables broadband hyperspectral imaging with quantitative phase, absorption, and dispersion information from 240 to $450 \mathrm{~nm}$. While the UHI system has access to more detailed spectral information compared with the simpler brightfield, multispectral UV microscope system, the UHI system is bulkier, and can be far more challenging to align (owing to the low spatial and temporal coherence of the source). Therefore, each method has its unique strengths with UHI being more appropriate for research, whereas the brightfield, multispectral UV system is more apt for clinical/ translational applications.

The optical density $(\mathrm{OD}(x, y))$ of a UV-transmission image at a specific $x$ - $y$ location depends on the measured intensity $\left(I_{\mathrm{m}}(x, y)\right)$ at that location, and the intensity of a blank field $\left(I_{0}(x, y)\right)$ according to the Beer-Lambert law. ${ }^{10,20}$ The blank field is obtained by capturing an image without the sample, keeping all other imaging conditions (e.g., camera's integration time) unchanged (this compensates for any system or detector spectral dependence). The OD is thus defined as

$$
\mathrm{OD}(x, y)=-\log _{10}\left(\frac{I_{m(x, y)}}{I_{0}(x, y)}\right) .
$$

Here, we take the OD to be equal to the absorbance. ${ }^{20}$ The typical OD values for RBCs are in the approximate range 0.69 to 0.81 at $220 \mathrm{~nm}, 0.1$ to 0.22 at $260 \mathrm{~nm}, 0.12$ to 0.24 at $280 \mathrm{~nm}$, and 0.03 to 0.15 at $300 \mathrm{~nm}$. The absorption at 220, 260, and $280 \mathrm{~nm}$ is high from both $\mathrm{Hb}$ and other membrane proteins. Thus, an effective molar extinction coefficient for RBCs can be calculated using a weighted average of the extinction coefficients for $\mathrm{Hb}^{29}(95 \%)$ and an "average protein" $20,22(5 \%)$ (whose extinction coefficient is calculated using the procedure detailed in Ref. 20; deviations in this value will have minimal impact on the overall mass). Although the $\mathrm{Hb}$ content may vary between $95 \%$ and $98 \%$ of the dry mass, this small variation will have a proportionally small effect $(\leq 3 \%)$ on the $\mathrm{Hb}$ mass estimate. As reported in Ref. 22 , the effective $\varepsilon$ values used for RBCs are: $\varepsilon_{220}=606,000 \mathrm{Lmol}^{-1} \mathrm{~cm}^{-1}, \varepsilon_{260}=112,360 \mathrm{Lmol}^{-1} \mathrm{~cm}^{-1}$, and $\varepsilon_{280}=115,634 \mathrm{Lmol}^{-1} \mathrm{~cm}^{-1}$. A detailed explanation of the calculation of these effective values is provided in the Supplemental Material. At $300 \mathrm{~nm}$, we assume that $\mathrm{Hb}$ is the only significant absorber and that contributions from the membrane proteins are negligible, and hence $\varepsilon_{300}=\varepsilon_{\mathrm{Hb}_{300}}=65,972 \mathrm{Lmol}^{-1} \mathrm{~cm}^{-1}$. Hence, the dry mass per unit area $(\sigma)$ of the RBC is given as

$$
\sigma_{\mathrm{RBC}_{\lambda}}(x, y)=\frac{\mathrm{OD}(x, y)}{\varepsilon_{\lambda}} M,
$$

where $M$ is the molar mass of the constituents. Based on the previous assumptions, at 220, 260, and $280 \mathrm{~nm}$, the dry mass of $\mathrm{Hb}$ is $95 \%$ of the total dry mass obtained from absorption-based 
measurements, i.e., $\sigma_{\mathrm{Hb}_{\lambda}}=0.95 \sigma_{\mathrm{RBC}_{\lambda}}$. Similarly, $M$ in Eq. (2) is $63,911 \mathrm{~g} / \mathrm{mol}$ a weighted average of the molar masses of hemoglobin $(64,500 \mathrm{~g} / \mathrm{mol})$ and the "average" protein $(52,728 \mathrm{~g} / \mathrm{mol})$. At $300 \mathrm{~nm}$, since all the absorption is assumed to be from $\mathrm{Hb}$, we have $\sigma_{\mathrm{Hb}_{\lambda}}=$ $\sigma_{\mathrm{RBC}_{\lambda}}$ and $M=M_{H b}=64,500 \mathrm{~g} / \mathrm{mol}$.

A similar set of assumptions needs to be made for mass mapping with QPI. The phase accumulated as light travels through a sample depends on the wavenumber, the difference between the refractive indices of the sample and surroundings, and the sample thickness. The refractive index of a cell at a given wavelength $\left(n_{\text {cell }}(\lambda)\right)$ is related to its concentration $(c)$ as $n_{\text {cell }}(\lambda)=n_{m}(\lambda)+c \beta_{\lambda}$, where $\beta_{\lambda}$ is the refractive index increment at a specific wavelength and $n_{m}$ is the refractive index of the surrounding medium, which is considered to be very similar to the refractive index of the cytoplasm, for cells suspended in media. ${ }^{5}$ Therefore, the dry mass per unit area is related to the phase via

$$
\sigma_{\lambda}(x, y)=\frac{\lambda}{2 \pi \beta_{\lambda}} \phi_{\lambda}(x, y)
$$

where $\phi$ is the measured phase. ${ }^{19,30}$ Here, we follow established QPI-based methods for assessing RBCs mass maps, which do not consider contributions from proteins. ${ }^{6}$ Accordingly, we use refractive index increments for $\mathrm{Hb}$ reported in Ref. 26, averaged over a 10-nm bandwidth. Hence, we have $\beta_{260}=0.2561 \mathrm{~mL} / \mathrm{g}, \beta_{280}=0.2304 \mathrm{~mL} / \mathrm{g}$, and $\beta_{300}=0.2183 \mathrm{~mL} / \mathrm{g} .{ }^{26}$ The total mass of the cell (from both methods) is computed from the mass density as

$$
\operatorname{mass}=\sum_{x} \sum_{y} \sigma_{\lambda}(x, y) \cdot p s,
$$

where $p s$ is the pixel size and the $x$ and $y$ coordinates cover the entire cell area.

\section{Results and Discussion}

For UV absorption-based mass mapping, whole blood is collected from a healthy donor according to protocols approved by the Institutional Review Board of Georgia Institute of Technology and Emory University. A blood smear is prepared and imaged, after air drying for $5 \mathrm{~min}$, at the four different wavelengths using the multispectral UV microscopy setup. The intensity images at each wavelength, normalized by the blank reference, are coregistered using a simple intensitybased registration algorithm implemented in MATLAB (Mathworks). The mass map is calculated at each wavelength using Eq. (2). Unphysical values of mass per area, such as negative values, are thresholded. The intensity stack at the four wavelengths and the associated mass map at $280 \mathrm{~nm}$ are shown in Fig. 1(a), where the high absorption at $220 \mathrm{~nm}$ compared to the other wavelengths can be clearly seen (cells appear very dark in the grayscale intensity maps).

To analyze individual RBCs, we identify rectangular regions of interest (ROIs) containing representative cells $(N=36)$ that have minimal to no overlap with surrounding cells. Then, the RBCs are further segmented using Otsu's thresholding method, followed by morphological operations including erosion, dilation, opening, and closing using a disk element to refine the mask and avoid edge (diffraction) artifacts arising from some degree of spatial coherence of the light source ${ }^{31}$ and wavelength-dependent aberrations. This segmentation is repeated for the same set of cells at all four wavelengths and yields unique masks at each wavelength, which accounts for slight variations in the images (e.g., from wavelength-dependent aberrations). A fully automated analysis pipeline that uses more sophisticated algorithms would greatly improve our throughput and will form part of our future work.

The mass maps of a typical healthy RBC at the four wavelengths are shown in Fig. 1(b). While they are generally similar, small variations in morphological features are possibly due to a combination of aberrations, lensing effects, ${ }^{32,33}$ and diffraction artifacts. At $220 \mathrm{~nm}$, strong absorption from both membrane proteins and $\mathrm{Hb}$ results in a homogeneous mass map with a high contrast-to-noise ratio (CNR). The mass maps at 260 and $280 \mathrm{~nm}$ are very similar, but mass maps extracted from $280 \mathrm{~nm}$ illumination are slightly more uniform because of the relatively higher absorption from proteins and $\mathrm{Hb}$ at $280 \mathrm{~nm}$ compared to $260 \mathrm{~nm}$. At $300 \mathrm{~nm}, \mathrm{Hb}$ 
(a)

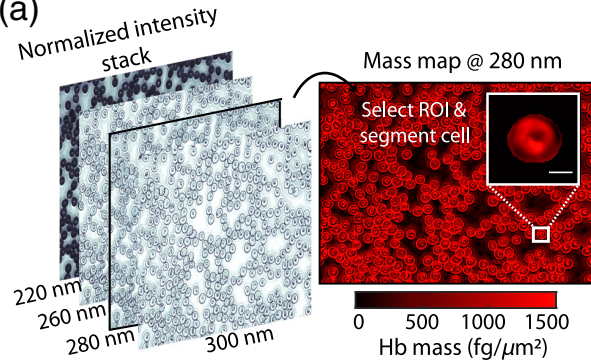

(c)

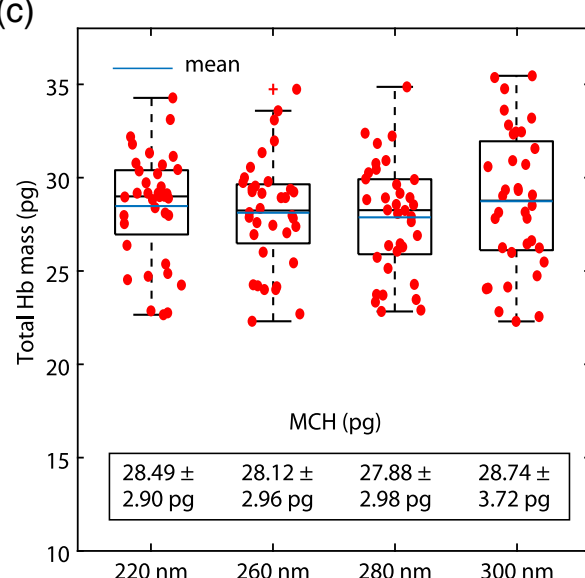

(b)

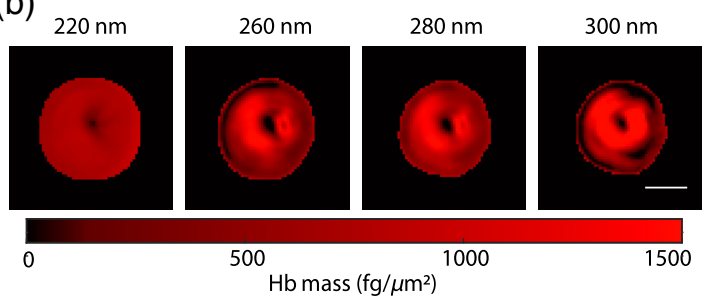

(d)

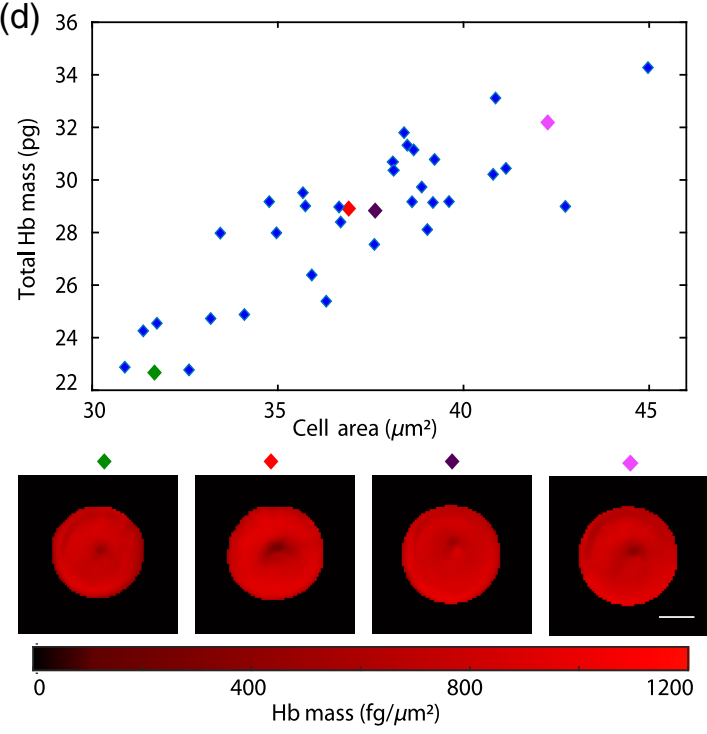

Fig. 1 (a) UV absorption-based mass mapping workflow. A mass map is generated corresponding to each normalized and coregistered intensity in the stack, followed by the selection of a rectangular ROI and cell segmentation. (b) Mass maps at 220, 260, 280, and $300 \mathrm{~nm}$ from a typical RBC. (c) Box plot showing distributions of $36 \mathrm{RBCs}$ at $220,260,280$, and $300 \mathrm{~nm}$. MCH values are listed on the plot. (d) Distribution of total masses with cell area at $220 \mathrm{~nm}$. Example cells are shown below the plot. (Scale bars: $5 \mu \mathrm{m}$ ).

absorption is the lowest of the four wavelengths under consideration, and protein absorption is negligible, resulting in a noisier mass map (i.e., a worse CNR).

The boxplot in Fig. 1(c) shows the distributions of the total cell masses at the four wavelengths obtained using Eqs. (2) and (4) (the pixel size is $0.0216 \mu \mathrm{m} .{ }^{2}$ ). At 220, 260, and $280 \mathrm{~nm}$, the total masses have comparable ranges, mean, and median values. The MCH (total dry mass averaged across all the cells) is the highest at $300 \mathrm{~nm}(28.74 \mathrm{pg})$ and is the lowest at $280 \mathrm{~nm}$ $(27.88 \mathrm{pg}$ ), with no statistically significant differences between the four wavelengths (using a two-tailed $t$-test). The standard deviation of the $\mathrm{Hb}$ dry mass is lowest at $220 \mathrm{~nm}(2.90 \mathrm{pg})$, very similar at 260 and $280 \mathrm{~nm}$, and highest at $300 \mathrm{~nm}(3.72 \mathrm{pg})$, yielding a coefficient of variation $(\mathrm{CV}) \sim 10 \%$. The $\mathrm{CV}$ of the $\mathrm{Hb}$ mass distribution may be assumed to be equivalent to the red cell distribution width (based on correlation analyses in Ref. 23 ), which is typically $11 \%$ to $16 \% .^{34}$ Since we consider a relatively small number of cells, we may be unable to capture the variation across the entire patient RBC population, and the difference in the standard deviations of the $\mathrm{Hb}$ masses at different wavelengths may be due to broadening of the $\mathrm{Hb}$ mass distribution due to measurement errors. The total cell mass is plotted against the cell area in Fig. 1(d), and we observe that both the areas and masses are within the range of values for healthy RBCs. ${ }^{22,24}$ Mass maps from certain representative cells of different sizes are shown below the plot.

The UV absorption-based mass mapping method is directly dependent on the accuracy of the extinction coefficient values applied in the calculations. For $\mathrm{Hb}$ absorption, $300 \mathrm{~nm}$ is an isosbestic point (absorption is approximately equal for all forms of $\mathrm{Hb}$ ), whereas the molar extinction coefficient at other wavelengths varies with oxygenation. Furthermore, while there is significant absorption from proteins at 220,260 , and $280 \mathrm{~nm}$, the absorption at $300 \mathrm{~nm}$ is 
dominated by $\mathrm{Hb}$ alone, leading to the expectation that $\mathrm{Hb}$-quantification at $300 \mathrm{~nm}$ would be most precise. ${ }^{24}$ The absorption coefficient at $220 \mathrm{~nm}$ has not been accurately measured or widely reported, and here we adopted the value determined empirically in Ref. 22. Despite these shortcomings, we find that the $\mathrm{Hb}$ masses at $220 \mathrm{~nm}$ have high CNR and have the lowest standard deviation (i.e., highest precision). Similar results are also obtained at 260 and $280 \mathrm{~nm}$. On the contrary, because of the lower CNR, mass mapping at $300 \mathrm{~nm}$ performs the worst, defying previous expectations. Theoretically, it is possible to combine the measurements at different wavelengths to obtain robust estimates of the mass of $\mathrm{Hb}$ and non- $\mathrm{Hb}$ proteins, but this is hindered by imperfect pixelwise registration and wavelength-dependent aberrations. Moreover, the focus of this work is on $\mathrm{Hb}$ quantification using single-wavelength measurements (here our baseline ground truth is based on established QPI methods). UV microscopy images acquired at $260 \mathrm{~nm}$ have inherent nuclear contrast due to the absorption peak of nucleic acids and can capture all the features necessary for a five-part WBC differential. ${ }^{24}$ The results presented in this work demonstrate that the $\mathrm{Hb}$ quantification of RBCs at $260 \mathrm{~nm}$ is precise and reliable. These results are significant because they indicate that label-free hematology analysis can be achieved using only a single wavelength, which can lead to the development of a faster, more compact, and cost-effective point-of-care hematology analyzer.

Next, we compare the results obtained with UV absorption-based mass mapping to QPI-based mass mapping, which is a more well-established approach for dry mass mapping. ${ }^{5,6,17-19}$ To this end, isolated RBCs suspended in PBS are imaged with our UHI setup at a center wavelength of $275 \mathrm{~nm}$, which gives us access to a spectral range of $\sim 250$ to $325 \mathrm{~nm}$. The resultant hyperspectral data-cube is processed to produce hyperspectral phase images. ${ }^{26}$ The phase images of $15 \mathrm{RBCs}$ are averaged over a 10-nm bandwidth with center wavelengths 260 , 280, and $300 \mathrm{~nm}$ to mimic the effect of the bandpass filters in our multispectral setup and ensure fair comparisons. Due to the limited throughput of the interferometric configuration, data at $220 \mathrm{~nm}$ are not available for comparison. The phase images at each wavelength are segmented using Otsu's thresholding method, followed by morphological operations (described previously). This segmentation process provides the same treatment as with the UV absorption-based maps above. The total mass is obtained from the segmented mass map using Eq. (4) with a pixel size of $0.1 \mu \mathrm{m}^{2}$. The distributions of the total masses at each wavelength are shown by the boxplots in Fig. 2(a). The MCH is $\sim 28$ pg (within the healthy reference range ${ }^{7}$ ), with statistically insignificant variations across wavelength. Similarly, the standard deviations of the single-cell $\mathrm{Hb}$ mass as measured across all wavelengths are $\sim 4 \mathrm{pg}$, with statistically insignificant variations across wavelength. Some example mass maps are shown in Fig. 2(b), which clearly highlight the typically biconcave morphology of healthy RBCs. The mass maps at the different wavelengths have nearly identical morphology, and differences in the masses at different wavelengths likely arise from noise fluctuations at each wavelength.

(a)

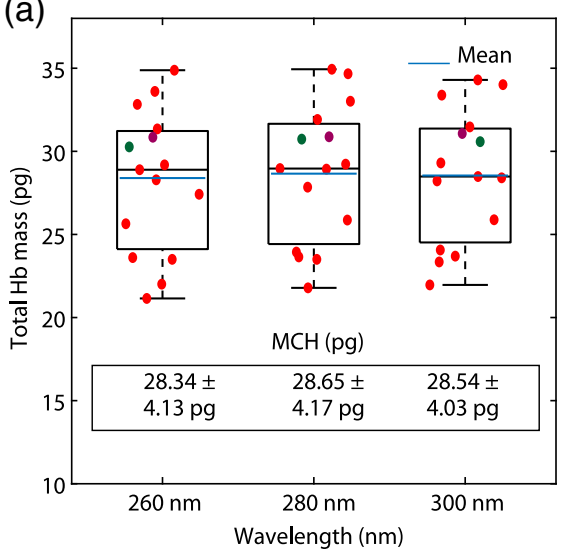

(b)

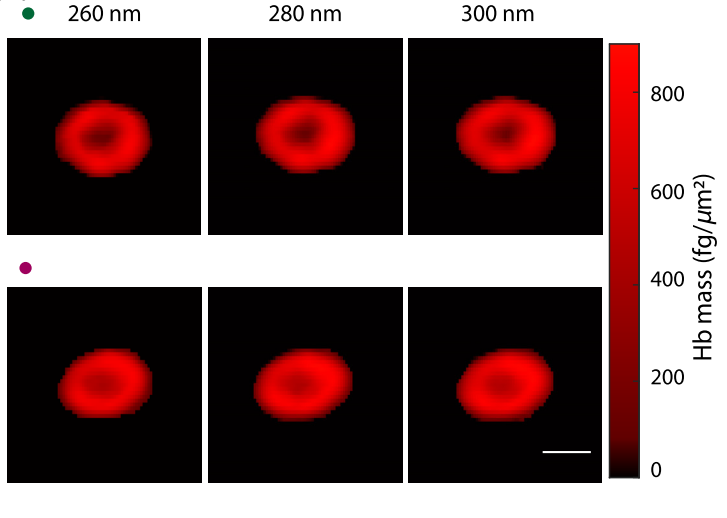

Fig. 2 (a) QPI-based mass mapping. (a) Box plot showing distributions of 15 RBCs at 260, 280, and $300 \mathrm{~nm}$. MCH values are listed on the plot. (b) Example mass maps of typical healthy RBCs (Scale bar: $5 \mu \mathrm{m}$ ). 
(a)

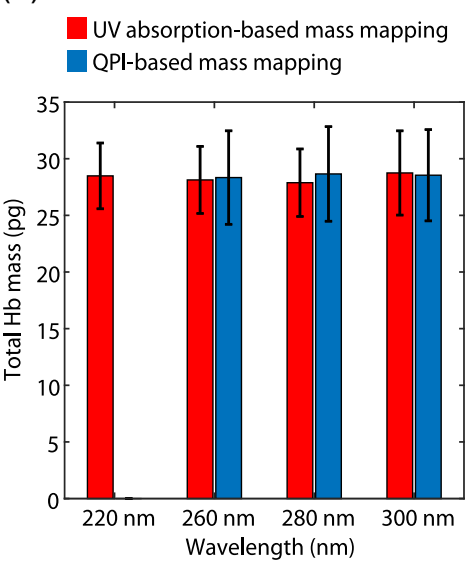

(b)
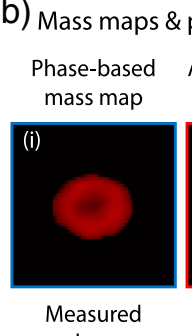

phase

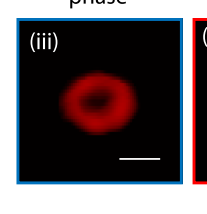

Cell imaged (c) Mass maps \& phases @ $280 \mathrm{~nm}$

Phase-based Absorption-based mass map mass map

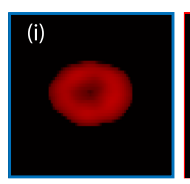

Measured

phase
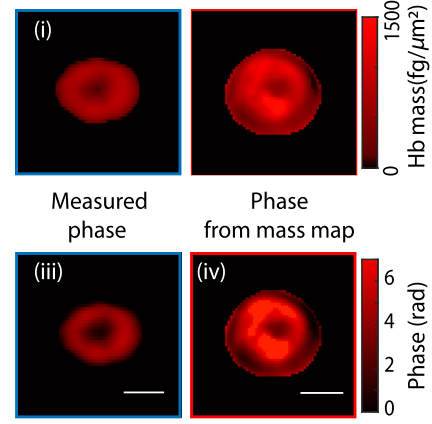

Phase

from mass map

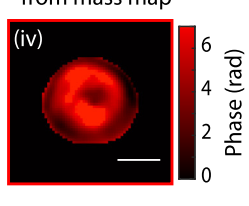

Fig. 3 (a) Comparison of $\mathrm{Hb}$ mass from both mass mapping methods. (b) Phase maps obtained from mass maps of a cell imaged with the bright-field UV system compared with QPI-based mass maps and phases obtained using the UHI system of a similar cell at $260 \mathrm{~nm}$. (c) Phase maps obtained from mass maps of a cell imaged with the brightfield UV system compared with QPIbased mass maps and phases obtained using the UHI system of a similar cell at $280 \mathrm{~nm}$ : (i) QPI-based mass map, (ii) UV absorption-based mass map, (iii) phase from UHI data, and (iv) phase from UV absorption-based mass map. (Scale bars: $5 \mu \mathrm{m}$ ).

The MCH values obtained from the two methods (referred to as UV and QPI henceforth) are compared in Fig. 3(a) and are in excellent agreement at 260, 280, and $300 \mathrm{~nm}$ (QPI-based data is not available at $200 \mathrm{~nm}$ ). While no ground truth is available for the $\mathrm{MCH}$ (since a CBC is not performed for a blood sample from a healthy donor), we take the agreement between the $\mathrm{MCH}$ values obtained with both methods and the well-accepted healthy $\mathrm{MCH}$ reference range as an indication that our measurements are producing realistic results. The dry mass measurements from UV absorption-based mass mapping have a lower standard deviation (2.96 versus $4.13 \mathrm{pg}$ at $260 \mathrm{~nm}$ ) compared to the QPI-based method. This discrepancy may be a result of the biological variation (as different cells are imaged with both methods), and the smaller available sample size for the QPI-based method. Notwithstanding, the UV absorption-based mass maps have a higher spatial resolution owing to the noninterferometric, intensity-based imaging system configuration compared to the UHI system, which may contribute to an improved precision in $\mathrm{Hb}$ mass mapping. We note that this advantage will persist for our bright-field, intensity-based UV system versus more conventional QPI systems, which operate in the visible spectral range. While a high spatial resolution is not critical to estimate the total $\mathrm{Hb}$ mass of a cell, the improved spatial resolution of morphological images and mass density maps may be beneficial for disease diagnosis ${ }^{5,6}$ or aid in the study of RBC dynamics. ${ }^{35}$

Finally, given the linear relationship between optical phase and dry mass [as described by Eq. (3)], intensity-based UV mass maps may be translated to optical phase. To this end, we use the mass maps at a specific UV wavelengths, along with the $\beta$ value at that those wavelengths (known a priori), to deduce optical phase information, following Eqs. (2) and (3). Figures 3(b) and 3(c) (ii and iv) show representative mass maps and phases (as derived from their initial UV mass estimate by effectively simulating the cells in PBS) at 260 and $280 \mathrm{~nm}$ for the same cell. Similarly, the mass maps obtained from phase measurements for a different cell imaged with UHI microscopy are shown for comparison in Figs. 3(b) and 3(c) (i and iii). The phase measurements reflect the expected biconcave shape of the cells at both wavelengths. We note that the images from the two systems have slightly different resolutions and magnification, giving rise to some of the observed differences. The phase values from the UV absorption-based mass maps do appear slightly higher, which could be largely due to biological variation. In addition, changes in the mass distribution of $\mathrm{Hb}$ in the smears as compared to the cells in media as well as inaccuracies in the optical constants used to calculate the phases could contribute to the differences in the observed phase values.

In conclusion, we have presented UV absorption-based mass mapping of individual RBCs and $\mathrm{Hb}$ quantification at four wavelengths: $220,260,280$, and $300 \mathrm{~nm}$. The estimated MCH 
values at the different wavelengths show no statistically significant differences, and the standard deviations of the mass measurements are nearly identical at 220, 260, and 280 but slightly higher at $300 \mathrm{~nm}$. These results are surprising given that (1) $300 \mathrm{~nm}$ is an isosbestic point for $\mathrm{Hb}$, which one would expect to remove potential variability in the extinction coefficient due to oxygenation levels, and (2) there are no other significant absorbers at this wavelength. Our data, however, indicate that the lower absorption at $300 \mathrm{~nm}$ leads to an overall lower CNR and hence a less precise measurement of $\mathrm{Hb}$ content. In addition, despite potential variability in oxygenation and contributions from other absorbers, RBC and $\mathrm{Hb}$ characterization at 220, 260, $280 \mathrm{~nm}$ provide more precise results. Further, in this work, we compared our results to QPI-based mass mapping, which is well accepted, and found excellent agreement on average between the two methods. Finally, we have demonstrated that our UV absorption-based mass maps can be translated into phase maps, from which other phase-related metrics can be computed. Therefore, deep-UV microscopy enables high-resolution, label-free morphological imaging, and accurate mass mapping and $\mathrm{Hb}$ quantification. Importantly, deep-UV microscopy images acquired at a single-wavelength $(260 \mathrm{~nm})$ can be used for characterizing RBCs and WBCs, thus enabling fast and costeffective hematology analysis that can result in a simpler, point-of-care hematology analyzer.

\section{Disclosures}

The authors have no conflicts of interest to disclose.

\section{Acknowledgments}

We greatly acknowledge support for this work by the Massner Lane Family Foundation; Burroughs Wellcome Fund (CASI BWF 1014540); National Science Foundation (NSF CBET CAREER 1752011); and the Donaldson Charitable Trust Research Synergy Fund Award, a philanthropic award provided by the Winship Cancer Institute of Emory University, the Aflac Cancer \& Blood Disorders Center at Children's Healthcare of Atlanta, and the Wallace H. Coulter Biomedical Engineering Department at Emory University and the Georgia Institute of Technology.

\section{References}

1. T. Honda et al., "Neutrophil left shift and white blood cell count as markers of bacterial infection," Clin. Chim. Acta 457, 46-53 (2016).

2. T. B. Newman et al., "Combining immature and total neutrophil counts to predict early onset sepsis in term and late preterm newborns: use of the I/T2," Pediatr. Infectious Disease J. 33, 798-802 (2014).

3. J. D. Seebach et al., "The diagnostic value of the neutrophil left shift in predicting inflammatory and infectious disease," Am. J. Clin. Pathol. 107, 582-591 (1997).

4. M. S. Park et al., "Leukopenia predicts remission in patients with inflammatory bowel disease and behcet's disease on thiopurine maintenance," Digestive Diseases Sci. 60, 195-204 (2015).

5. H. Majeed et al., "Quantitative phase imaging for medical diagnosis," J. Biophotonics 10(2), 177-205 (2017).

6. M. Mir, K. Tangella, and G. Popescu, "Blood testing at the single cell level using quantitative phase and amplitude microscopy," Biomed. Opt. Express 2(12), 3259 (2011).

7. S. Lewis, B. Bain, and I. Bates, Dacie and Lewis Practical Haematology, 10th ed., Churchill Livingstone/Elsevier, Philadelphia (2006).

8. R. Barer, "Refractometry and interferometry of living cells," J. Opt. Soc. Am. 47, 545-556 (1957).

9. R. I. Weed, C. F. Reed, and G. Berg, "Is hemoglobin an essential structural component of human erythrocyte membranes?" J. Clin. Invest. 42(4), 581-588 (1963).

10. M. C. Cheung et al., "Deep ultraviolet mapping of intracellular protein and nucleic acid in femtograms per pixel," Cytom. Part A 79A(11), 920-932 (2011). 
11. J. M. Higgins and L. Mahadevan, "Physiological and pathological population dynamics of circulating human red blood cells," Proc. Natl. Acad. Sci. U. S. A. 107(47), 20587-20592 (2010).

12. D. J. Weatherall, "Systems biology and red cells," N. Engl. J. Med. 364, 376-377 (2011).

13. D. H. Tycko et al., "Flow-cytometric light scattering measurement of red blood cell volume and hemoglobin concentration," Appl. Opt. 24, 1355-1365 (1985).

14. Y. R. Kim and L. Ornstein, "Isovolumetric sphering of erythrocytes for more accurate and precise cell volume measurement by flow cytometry," Cytometry 3(6), 419-427 (1983).

15. E. Schonbrun et al., "Quantitative absorption cytometry for measuring red blood cell hemoglobin mass and volume," Cytom. Part A 85, 332-338 (2014).

16. M. H. Steinberg et al., Disorders of Hemoglobin: Genetics, Pathophysiology, and Clinical Management, 2nd ed., Cambridge University Press, Cambridge (2009).

17. R. Barer, "Interference microscopy and mass determination," Nature 169, 366-367 (1952).

18. H. Davies and E. Deeley, "An integrator for measuring the 'dry mass' of cells and isolated components," Exp. Cell. Res. 11(1), 169-185 (1956).

19. G. Popescu et al., "Optical imaging of cell mass and growth dynamics," Am. J. Physiol. Cell Physiol. 295 (2), C538-C544 (2008).

20. B. J. Zeskind et al., "Nucleic acid and protein mass mapping by live-cell deep-ultraviolet microscopy," Nat. Methods 4(7), 567-569 (2007).

21. Y. Park et al., "Spectroscopic phase microscopy for quantifying hemoglobin concentrations in intact red blood cells," Opt. Lett. 34, 3668-3670 (2009).

22. M. C. Cheung et al., "Intracellular protein and nucleic acid measured in eight cell types using deep-ultraviolet mass mapping," Cytom. Part A 83A, 540-551 (2013).

23. Y. Kim et al., "Profiling individual human red blood cells using common-path diffraction optical tomography," Sci. Rep. 4, 6659 (2014).

24. A. Ojaghi et al., "Label-free hematology analysis using deep-ultraviolet microscopy," Proc. Natl. Acad. Sci. U. S. A. 117(26), 14779-14789 (2020).

25. M. E. Kandel et al., "Phase imaging with computational specificity (pics) for measuring dry mass changes in sub-cellular compartments," Nat. Commun. 11, 6256 (2020).

26. A. Ojaghi et al., "Ultraviolet hyperspectral interferometric microscopy," Sci. Rep. 8(1), 1-6 (2018).

27. S. Soltani, A. Ojaghi, and F. E. Robles, "Deep UV dispersion and absorption spectroscopy of biomolecules," Biomed. Opt. Express 10(2), 487 (2019).

28. N. Kaza, A. Ojaghi, and F. E. Robles, "Ultraviolet hyperspectral microscopy using chromatic-aberration-based iterative phase recovery," Opt. Lett. 45, 2708-2711 (2020).

29. S. Prahl, "Optical absorption of hemoglobin," https://omlc.org/spectra/hemoglobin/index .html.

30. T. A. Zangle and M. A. Teitell, "Live-cell mass profiling: an emerging approach in quantitative biophysics," Nat. Methods 11, 1221-1228 (2014).

31. P. Chieco et al., "A user's guide for avoiding errors in absorbance image cytometry: a review with original experimental observations," Histochem. J. 26, 1-19 (1994).

32. L. Miccio et al., "Red blood cell as an adaptive optofluidic microlens," Nat. Commun. 6, 6502 (2015).

33. P. Memmolo et al., "The talbot effect in self-assembled red blood cells investigated by digital holography," J. Phys. Photonics 2, 035005 (2020).

34. J. E. May et al., "Three neglected numbers in the CBC: the RDW, MPV, and NRBC count," Cleveland Clinic Journal of Medicine 86(3), 167-172 (2019).

35. G. Popescu et al., "Imaging red blood cell dynamics by quantitative phase microscopy," Blood Cells Mol. Dis. 41(1), 10-16 (2008).

36. A. T. Francis et al., "In vitro quantification of single red blood cell oxygen saturation by femtosecond transient absorption microscopy," J. Phys. Chem. Lett. 10(12), 3312-3317 (2019).

37. Y. Sugita, M. Nagai, and Y. Yoneyama, "Circular dichroism of hemoglobin in relation to the structure surrounding the heme," J. Biol. Chem. 246(2), 383-388 (1971). 
Nischita Kaza is a PhD student in the bioengineering program through the School of Electrical and Computer Engineering at Georgia Tech. She received her undergraduate degree in electrical and electronics engineering at the National Institute of Technology, Surathkal, India. Her research interests lie in label-free imaging, image processing, and machine learning for biomedical data analysis.

Ashkan Ojaghi is a $\mathrm{PhD}$ student in the Wallace H. Coulter Department of Biomedical Engineering at Georgia Tech \& Emory University. He received his MSc degree from the Department of Mechanical Engineering at York University, Canada, in 2016, and his BSc degree in mechanical engineering from the Iran University of Science and Technology in 2014. Currently, he is conducting research on deep-UV microscopy and spectroscopy of biological samples.

Francisco E. Robles is an assistant professor at the Wallace H. Coulter Department of Biomedical Engineering at Georgia Tech \& Emory University. His lab focuses on advancing label-free optical imaging technologies to help improve our understanding of biological processes and our ability to identify disease. He earned his doctorate in medical physics at Duke University and completed his postdoctoral training in the Department of Chemistry also at Duke. 